\title{
Ventilator-associated Pneumonia
}

\author{
Harprit Kaur Madan
}

\begin{abstract}
Ventilator-associated pneumonia (VAP) is the most frequent intensivecare unit (ICU)-acquired infection for patients on mechanical ventilators. There are no goal standards for VAP diagnosis and this makes it the leading cause of death in critically ill patients. The prediction of its outcome is important in the decision-making process and management. This article reviews the various aspects of VAP such as definition, risk factors, etiological agents, diagnosis, treatment, and prevention with emphasis on the recent advances.
\end{abstract}

Keywords: Aspiration, Intubation, Mechanical ventilation, Ventilator-associated pneumonia.

Research and Innovation in Anesthesia (2019): 10.5005/jp-journals-10049-0075

\section{INTRODUCTION}

Ventilator-associated pneumonia (VAP) is a type of hospital-acquired pneumonia that develops after more than 48-72 hours after endotracheal intubation. It is the second most common nosocomial infection in hospital and is a leading cause of death in critically ill patients. It can also be conceptually defined as an inflammation of the lung parenchyma caused by infectious agents not present or incubating at the time mechanical ventilation (MV) was started.

Ventilator-associated pneumonia occurs in $9-24 \%$ of patients intubated for longer than 48 hours. Since it is clinically significant and one of the major challenges faced by intensivist in intensive care, VAP is studied as an individual clinical entity. Ventilatorassociated pneumonia is prolonging the length of stay at the ICU and increasing the risk of death in critically ill patients. ${ }^{1,2}$

\section{Classification}

- Early onset-onset of VAP occurs during the first 4 days of MV, usually less severe and associated with better prognosis. It is more likely to be caused by antibiotic-sensitive bacteria.

- Late onset-onset of VAP more than 96 hours after the initiation of mechanical ventilation. More commonly seen with multidrugresistant (MDR) pathogens and is associated with increased morbidity and mortality.

The CDC definitions ventilator-associated events (VAE-VACIVAC-VAP) $^{1,3}$

The CDC National Healthcare Safety Network implemented ventilator-associated events (VAE) surveillance in January 2013.

The VAE system is a three-tier surveillance definition to identify complications, including VAP in patients on mechanical ventilation $^{1,3}$ (Flowchart 1).

The ventilator-associated condition (VAC) - patients with a period of sustained respiratory deterioration (changes in PEEP $\geq 3 \mathrm{~cm}$ $\mathrm{H}_{2} \mathrm{O}$ or fraction of inspired oxygen $\left[\mathrm{FiO}_{2}\right] \geq 0.2$ for 2 days following a sustained period of stability or improvement on the ventilator).

Infection-related ventilator-associated complication (IVAC) patients with VAC with an abnormal temperature (below $36^{\circ} \mathrm{C}$ or above $\left.38^{\circ} \mathrm{C}\right)$ or white blood cell count $(\leq 4,000$ or $\geq 12,000$ cells/ $\mathrm{mm}^{3}$ ) and be started on one or more new antibiotics that continue for 4 or more days.

Possible and probable VAP — these are patients with IVAC and laboratory and/or microbiological evidence of respiratory infection.
Department of Anesthesiology and Critical Care, HinduHrudaySamrat Balasaheb Thackarey Medical College and Dr RN Cooper Municipal General Hospital, Mumbai, Maharashtra, India

Corresponding Author: Harprit Kaur Madan, Department of Anesthesiology and Critical Care, HinduHrudaySamrat Balasaheb Thackarey Medical College and Dr RN Cooper Municipal General Hospital, Mumbai, Maharashtra, India, Phone: +91 9820091317, e-mail: drpreet@hotmail.com

How to cite this article: Madan HK. Ventilator-associated Pneumonia. Res Inno in Anesth 2019;4(2):55-59.

Source of support: Nil

Conflict of interest: None

Possible VAP is when there is evidence of purulent pulmonary secretions or a pathogenic pulmonary culture on Gram stain in a patient with IVAC.

Probable VAP is when there is evidence of Gram stain purulence plus quantitative or semiquantitative growth of a pathogenic organism beyond specified thresholds.

\section{Clinical Features}

The average time taken to clinically develop VAP from the initiation of mechanical ventilation is around 5-7 days, with a mortality rate quoted as between $24 \%$ and $76 \%$. Most patients with VAP present with a gradual or sudden onset of the following signs and symptoms after 48 hours of MV. ${ }^{4}$

- Symptoms-dyspnea

- Signs-fever, tachypnea, increased or purulent secretions, hemoptysis, rhonchi, crackles, reduced breath sounds, bronchospasm

- Ventilator mechanics-reduced tidal volume, increased inspiratory pressures

- Laboratory findings-worsening hypoxemia, leukocytosis, inc. $C$ reactive protein (CRP)

- Imaging-new or progressive infiltrate on X-ray or CT scan

\section{Pathogenesis}

The main ${ }^{2,4,5}$ pathogenic factors in the development of VAP are the following: $2,4,5$

- Biofilm formation within the tracheal tube

(c) The Author(s). 2019 Open Access This article is distributed under the terms of the Creative Commons Attribution 4.0 International License (https://creativecommons. org/licenses/by-nc/4.0/), which permits unrestricted use, distribution, and non-commercial reproduction in any medium, provided you give appropriate credit to the original author(s) and the source, provide a link to the Creative Commons license, and indicate if changes were made. The Creative Commons Public Domain Dedication waiver (http://creativecommons.org/publicdomain/zero/1.0/) applies to the data made available in this article, unless otherwise stated. 
Flowchart 1: Ventilator-associated events

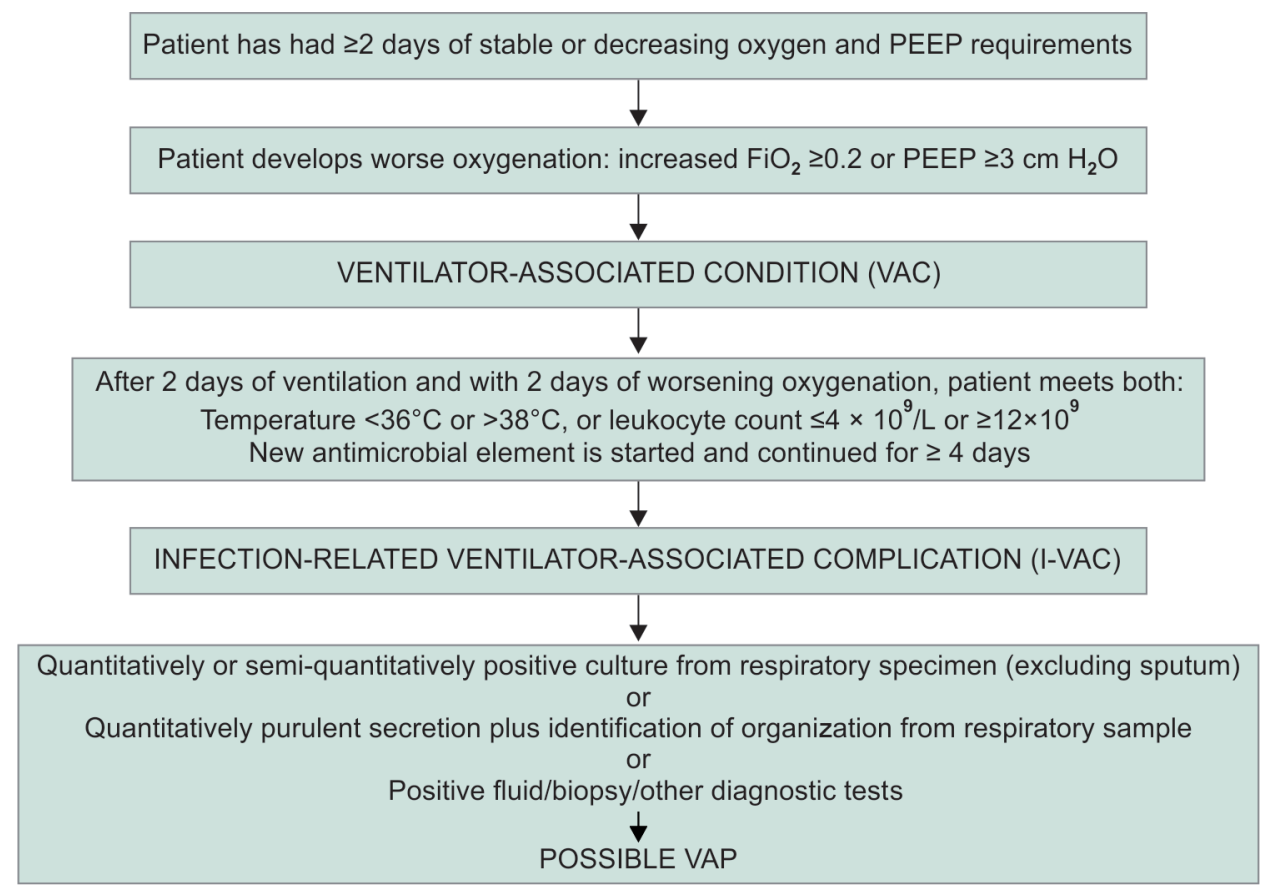

The endotracheal tube prevents normal coughing; the oropharynx becomes rapidly colonized by aerobic gram-negative bacteria and these secretions gain access to the lower airway. A bacterial biofilm, that is impervious to antibiotics, gradually develops on the inner surface of the endotracheal tube and acts as a nidus for infection. This pathogen-rich biofilm is then pushed into the distal airways by the ventilator cycling.

- Microaspiration of microbe laden secretions:

- Directly from oropharynx

- Gastric reflux

- Nursing patients in a supine position.

- Enteral feeding via a nasogastric tube

- Through a direct extension of a contiguous infection, e.g., pleural space infection

- Through inhalation of contaminated air or medical aerosols

- Increases the risk of aspiration of gastric contents; the longer the duration of ventilation, the greater the risk of developing VAP.

\section{RISK FACTORS $3,4,6$}

- Extreme age

- Chronic lung disease

- Aspiration/microaspiration from being nursed in a supine position

- Cardiac or thoracic surgery

- Reintubation after unsuccessful extubation or prolonged intubation
Contd...

- Previous antibiotic therapy, - Tracheostomy especially broad-spectrum antibiotics

- Acute respiratory distress syndrome

- Continuous sedation, use of paralytic agents

- Duration of the mechanical - Polytrauma patient ventilation

- Nasogastric tubes

- Burns

\section{Prevention}

Ventilator-associated pneumonia prolongs the duration of stay in the ICU, thus increasing the cost of patient management and increasing the ICU burden. Therefore, prevention of VAP is an utmost priority in the management of critical patients. Following are the methods to prevent VAP. ${ }^{2,7-9}$

\section{VAP: Reducing Time at Risk}

- Noninvasive positive pressure ventilation.

- Daily weaning trials and sedation holidays: Daily weaning trials and sedation holidays limit the time of mechanical ventilation.

- Reintubation is associated with a higher risk and incidence of VAP due to evidence of higher aspiration.

- Early tracheostomy.

\section{Preventing Aspiration and ETT Colonization}

- Cuff pressure control-an inflating cuff pressure $<20 \mathrm{~cm} \mathrm{H}_{2} \mathrm{O}$ favors increased passage of secretions between the cuff and the wall of the trachea, while $>30 \mathrm{~cm} \mathrm{H}_{2} \mathrm{O}$ may cause tracheal mucosal damage. Thus constantly monitor and adjust the ETT cuff inflation pressure.

- Cuff shape-surface of PVC ETT cuff folds when inflated in the trachea, creating potential channels through which secretions 


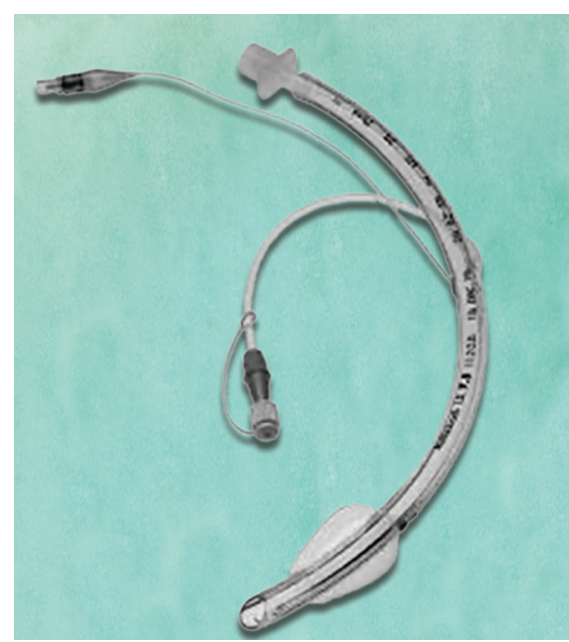

Fig. 1: Subglottic endotracheal tube with tapered cuff ETT

can enter the lower respiratory tract. By using ETT made of ultrathin polyurethane with the cuff in tapered shape protects against the secretion channeling below the cuff (Fig. 1).

- Head elevation-30-45 helps to reduce aspiration of gastric content.

- Subglottic suction ports-subglottic secretion drainage systems usually consist of an accessory aspiration conduit opening above the endotracheal tube cuff and a vacuum source (Fig. 2).

- Minimizing excessive time on a ventilator by implementation of an early weaning protocol with regular sedation breaks.

- Avoidance of routine or scheduled ventilator circuit changes.

- Stress ulcer prophylaxis.

- Reducing unplanned extubations and minimizing reintubationreintubation is associated with a higher risk of VAP due to higher rates of aspiration.

- ETT coating - bacterial colonization and biofilm formation on the inner surface of the tube can be prevented by coating it with a thin layer of antimicrobial agents.

- Gastric volume monitoring-monitor the patient's gastric residual volume at regular intervals and prior to increasing the infusion rate of gastric tube feeding, to minimize the risk of unrecognized gastric fluid accumulation and vomiting resulting in pneumonia.

\section{Reducing Colonization}

- Decontamination of the digestive tract by applying antiseptics, such as chlorhexidine in the oropharynx, and nonabsorbable antibiotics with broad-spectrum activity, which can be applied to the oropharynx [selective oropharyngeal decontamination (SOD)] or administered enterally [selective digestive-tract decontamination (SDD)]. This antibiotic therapy is used to eradicate potentially pathogenic microorganisms in oral, gastric, and intestinal flora. ${ }^{10}$

- Silver-coated ETTs due to its broad-spectrum antibiotic properties of silver reduces risk for the development of VAP.

- Nebulized gentamicin-prevents biofilm formation inside the ETT.

- Oral decontamination-oral care with chlorhexidine. Dental plaque contains multiple pathogens (S. aureus and P. aeruginosa) After 48 hours, normal oral flora of critically ill patients changes to more virulent gram-negative organisms.

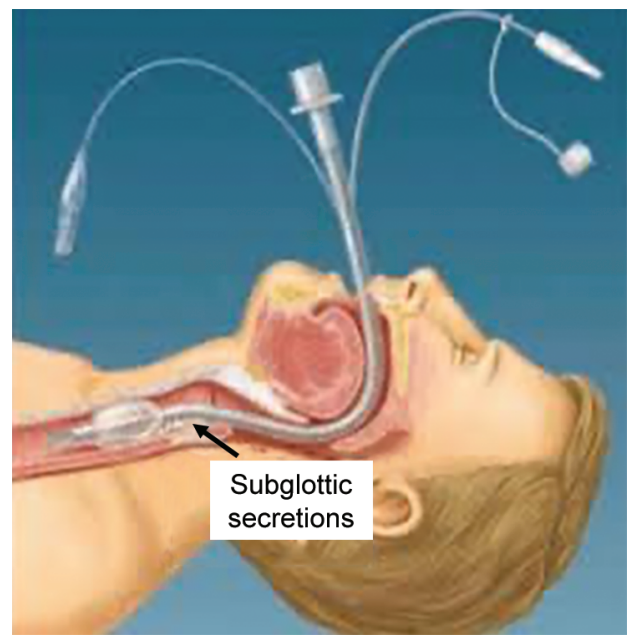

Fig. 2: Subglottic suctioning endotracheal tube in situ

- Probiotics-probiotics are living microorganisms with health benefit when administered in adequate dosages. Studies have shown that critically ill patients who received Lactobacillus rhamnosus had significantly fewer microbiologically confirmed cases of VAP and significantly fewer episodes of Clostridium difficile-associated diarrhea compared to patients who did not receive the probiotic.

- Use of heat and moisture exchanger filters.

- Feeding - gastric reflux and aspiration of gastric contents is one of the main causes of VAP. Post-pyloric feeding may reduce the incidence of VAP.

\section{Infection Control in the ICU}

- The aim of infection control is to prevent cross-transmission of pathogens, which plays an important role in the development of nosocomial infections including VAP. An effective strategy like education of the medical team, universal hand hygiene, use of personal protective equipment, and a protocol for microbiological surveillance helps in infection control.

- The VAP prevention bundles provide an effective method to reduce VAP.

\section{VAP Bundle}

A care bundle refers to a group of evidence-based interventions related to a particular condition, which when applied together significantly improves patient outcome.

- Elevation of the head of bed to between 30 and $45^{\circ}$

- Daily "sedation vacations" and assessment of readiness to extubate

- Peptic ulcer disease prophylaxis

- Deep venous thrombosis prophylaxis (unless contraindicated)

- Daily oral care with chlorhexidine

\section{Pathogens}

Early-onset VAP-more likely associated with non-multidrugresistant organisms E. coli, Klebsiella spp., Proteus spp., S. pneumoniae, H. influenzae, and S. aureus.

Late-onset VAP-more often associated with gramnegative bacilli, multidrug-resistant Pseudomonas aeruginosa, 
methicillin-resistant Staphylococcus aureus (MRSA), Enterobacter species, Acinetobacter spp., and Pseudomonas aeruginosa. 1,2,6,8

\section{DiAgnOSIS OF VAP}

Ventilator-associated pneumonia is diagnosed when a patient who has been mechanically ventilated for $\geq 48$ hours develops a new or progressive infiltrate with associated signs and symptoms of infection in whom positive respiratory specimens are present. Delayed diagnosis and delay in initiating antibiotic therapy is associated with worse outcomes in patients with VAP. ${ }^{1-3,9,11}$

\section{Clinical}

- Temperature $>38^{\circ} \mathrm{C}$

- Leukocytosis (WBC count $\geq 12,000$ cells $/ \mathrm{mm}^{3}$ ) or leukopenia (WBC count $<4,000$ cells $/ \mathrm{mm}^{3}$ )

- Presence of purulent secretions (purulent defined as $\geq 25$ neutrophils and $\leq 10$ squamous epithelial cells per low power field)

\section{Radiological}

Presence of newly developed infiltrates and/or consolidation.

\section{Microbiological}

As per the American Thoracic Society (ATS) and the Infectious Diseases Society of America (IDSA) guidelines, lower respiratory tract samples for culture and microbiology are recommended. Once specimens are obtained, they are sent for Gram stain, culture, and sensitivity. Culture results can be reported as semiquantitative and/or quantitative values.

Samples obtained by BAL techniques the diagnostic threshold is $10^{3}$ colony-forming units (CFU)/mL for protected specimen brushing and $10^{4} \mathrm{CFU} / \mathrm{mL}$ for BAL.

Bronchoscopic specimens-BAL or protected specimen brushing (PSB).

Nonbronchoscopic specimens-endotracheal aspirate, mini BAL.

Qualitative tracheobronchial aspirates are highly sensitive (N75\%) but poorly specific (b25\%) for the diagnosis of VAP.

Semiquantitative culture are cultures of tracheal secretions. They are considered positive when the agar growth is moderate $(+++)$ or heavy $(++++)$.

Quantitative cultures are generally preferred over qualitative culture for deciding the treatment for VAP. Quantitative positivity is defined as $\geq 10 \mathrm{CFU} / \mathrm{mL}$.

Blood and pleural fluid cultures - two sets of blood cultures and a thoracentesis for nonloculated pleural effusions of $\geq 10 \mathrm{~mm}$ in diameter on a lateral decubitus chest X-ray should be done for evaluation of suspected patients.

Urine routine and culture.

Biomarkers-Biomarkers are important diagnostic and prognostic markers of VAP.

CRP, procalcitonin (PCT), soluble triggering receptor expressed on myeloid cells-1 (sTREM-1), and endotoxin are the diagnostic biomarkers of VAP.

High levels of CRP are associated with poor outcome.

Low PCT levels in patients with no clinical signs of severe illness suggest deescalating of antibiotics.

Clinical pulmonary infection score (CPIS) - Pugin et al. developed a composite clinical score, called the CPIS, based on six variables: temperature, blood leukocyte count, volume
Table 1: CPIS scoring system

\begin{tabular}{|c|c|c|c|}
\hline CPIS points & 0 & 1 & 2 \\
\hline $\begin{array}{l}\text { Tracheal } \\
\text { secretions }\end{array}$ & Absent & Not purulent & $\begin{array}{l}\text { Abundant and } \\
\text { purulent }\end{array}$ \\
\hline $\begin{array}{l}\text { Leukocyte count } \\
\left(\mathrm{mm}^{3}\right)\end{array}$ & $\begin{array}{l}>4,000 \text { and } \\
<11,000\end{array}$ & $\begin{array}{l}<4,000 \text { and } \\
>11,000\end{array}$ & $\begin{array}{l}<4,000 \text { or } \\
>11,000\end{array}$ \\
\hline $\begin{array}{l}\text { Temperature } \\
\left({ }^{\circ} \mathrm{C}\right)\end{array}$ & $\begin{array}{l}>36.5 \text { and } \\
<38.4\end{array}$ & $\begin{array}{l}>38.5 \text { and } \\
<38.9\end{array}$ & $>39$ or $<36$ \\
\hline $\mathrm{PaO}_{2} / \mathrm{FiO}_{2}$ ratio & $>240$ or ARDS & - & $\begin{array}{l}<240 \text { and no } \\
\text { ARDS }\end{array}$ \\
\hline Chest X-ray & No infiltrate & $\begin{array}{l}\text { Diffuse } \\
\text { infiltrate }\end{array}$ & $\begin{array}{l}\text { Localized } \\
\text { infiltrate }\end{array}$ \\
\hline $\begin{array}{l}\text { Culture of } \\
\text { tracheal aspirate }\end{array}$ & Negative & - & Positive \\
\hline
\end{tabular}

and purulence of tracheal secretions, oxygenation, pulmonary radiography, and semiquantitative culture of tracheal aspirate. Scores can range between 0 and 12 with a score of $\geq 6$ showing good correlation with the presence of VAP.

A CPIS of $>6$ had a sensitivity of $93 \%$ and a specificity of $100 \%$ (Table 1).

\section{TREATMENT}

Late-onset VAP ( $>4$ days) requires broad-spectrum antibiotics whereas early-onset VAP ( $\leq 4$ days) can be treated with limitedspectrum antibiotics. Due to increased resistance, combination therapies are preferred over monotherapy.

Empirical treatment for VAP should include antibiotics with cover against Pseudomonas aeruginosa, Staphylococcus aureus, and gram-negative bacilli.

For empiric coverage for MRSA, either vancomycin or linezolid is recommended.

For MSSA regimen including piperacillin-tazobactam, cefepime, levofloxacin, imipenem, or meropenem.

Antipseudomonal carbapenem (imipenem or meropenem).

$\beta$-Lactam/ $\beta$-lactamase inhibitor (piperacillin-tazobactam).

For patients with VAP due to $P$. aeruginosa, the guidelines recommend that the choice of an antibiotic for definitive (not empiric) therapy be based on the results of antimicrobial susceptibility testing. For patients with VAP or HAP, a 7-day course of antimicrobial therapy is recommended. ${ }^{1-6,8}$

An updated antibiogram for each hospital and each ICU based on local bacteriological patterns is essential to guide initial empiric therapy. Delays in initiation of antibiotic treatment may lead to excess mortality from VAP. De-escalation is important to reduce emergence of resistance and should be done after microbiological culture results and the clinical response of the patient. The IDSA/ ATS guidelines highlight the importance of reassessing patients at 48-72 hours to determine whether the patient should continue antibiotic therapy for VAP or whether an alternative diagnosis should be pursued.

\section{CONCLUSION}

Ventilator-associated pneumonia is a major challenge to the intensivist with a high mobility and mortality and imposes significant financial burden on the healthcare system. It should be diagnosed based on a combination of different clinical and laboratory criteria. Most of the risk factors of VAP can be prevented. Ventilator-associated 
pneumonia is increasingly being associated with MDR pathogens like ESBL, AmpC $\beta$-lactamases, and metallo $\beta$-lactamases. The initial empiric therapy of VAP should be based on the presence or absence of risk factors for MDR pathogens. De-escalation of antibiotics is important to reduce emergence of resistance.

\section{References}

1. American Thoracic Society. Infectious diseases society of America. Guidelines for the management of adults with hospital-acquired, ventilator-associated, and healthcare-associated pneumonia. Am J Respir Crit Care Med 2005;171(4):388. DOI: 10.1164/rccm.200405644ST.

2. Gunasekera P, Gratrix A. Ventilator-associated pneumonia. BJA Educat 2016;16(6):198-202. DOI: 10.1093/bjaed/mkv046.

3. National Healthcare Safety Network (NHSN) July 2013 CDC/NHSN Protocol Clarifications 2013.

4. https://www.uptodate.com/contents/1635/.

5. Kalanuria AA, Zai W, Mirski M. Ventilator-associated pneumonia in the ICU. Crit Care 2014;18(2):208. DOI: https://doi.org/10.1186/cc13775.
6. Joseph NM, Sistla S, Dutta TK, et al. Ventilator-associated pneumonia: A review. Eur J Intern Med 2010;21(5):360-368. DOI: 10.1016/ j.ejim.2010.07.006.

7. Zolfaghari PS, Wyncoll DL. The tracheal tube: gateway to ventilatorassociated pneumonia. Crit Care 2011;15:310-317. DOI: 10.1186/ cc10352.

8. Koenig SM, Truwit JD. Ventilator-associated pneumonia: diagnosis, treatment, and prevention. Clin Microbiol Rev 2006;19(4):637-657. DOI: 10.1128/CMR.00051-05.

9. Berton DC, Kalil AC, Cavalcanti M, et al. Quantitative versus qualitative cultures of respiratory secretions for clinical outcomes in patients with ventilator-associated pneumonia. Cochrane Database Syst Rev 2012. CD006482. DOI: 10.1002/14651858.CD006482.pub3.

10. Silvestri L, Van Saene HKF, Casarin A, et al. Impact of selective decontamination of the digestive tract on carriage and infection due to gram-negative and gram-positive bacteria: a systematic review of randomised controlled trials. Anaesth Intensive Care 2008;36(3):324. DOI: $10.1177 / 0310057 X 0803600304$.

11. Meduri GU. Diagnosis and differential diagnosis of ventilatorassociated pneumonia. Clin Chest Med 1995;16(1):61. 